\title{
Instability of Rarefaction Shocks in Systems of Conservation Laws
}

\author{
J. A. Smoller, J. B. Temple \& Z. P. XIN \\ Communicated by C. DAFERMOS
}

\begin{abstract}
It is well-known that rarefaction shocks are unstable solutions of nonlinear hyperbolic conservation laws. Indeed, for scalar equations rarefaction shocks are unstable in the class of smooth solutions, but for systems one can only say in general that rarefaction shocks are unstable in the larger class of weak solutions. (Here unstable refers to a lack of continuous dependence upon perturbations of the initial data.) Since stability in the class of weak solutions is not well understood, ([T, TE]), "entropy" considerations have played a leading role in ruling out shocks that violate the laws of physics. However, for non-strictly hyperbolic systems the analogy with the equations of gas dynamics breaks down, and general entropy or admissibility criteria for the variety of shocks which appear, (see, e.g., [IMPT]), are not known. In this paper we address the question of when the instability of a shock can be demonstrated within the class of smooth solutions alone. We show by elementary constructions that this occurs whenever there exists an alternative solution to the Riemann problem with the same shock data which consists entirely of rarefaction waves and contact discontinuities with at least one non-zero rarefaction wave. We show that for $2 \times 2$ strictly hyperbolic, genuinely nonlinear systems the condition is both necessary and sufficient. We show too that for the full $3 \times 3$ (Euler) equations of gas dynamics with polytropic equations of state, rarefaction shocks of "moderate" strength are unstable in the class of smooth solutions if and only if the adiabatic gas constant $\gamma$ satisfies $1<\gamma<\frac{5}{3}$ (see Theorem 8). More precisely, there is a constant $y_{*}, 0<y_{*}<1$, depending only on $\gamma$, such that if $y_{*} p_{l} \leqq p_{r} \leqq p_{l}$ for 1 -shocks, and if $y_{*} p_{r} \leqq$ $p_{l} \leqq p_{r}$ for 3-shocks (where $p_{r}$ and $p_{l}$ denote the pressures on both sides of the rarefaction shock), then the shock is unstable if and only if $1<\gamma<\frac{5}{3}$. Thus for such shocks, the theory of the Riemann problem for polytropic gases in the range $1<\gamma<\frac{5}{3}$ can be rigorously developed with a knowledge of the smooth solutions alone by using stability under smoothing as an admissibility criterion, rather than by using the classical entropy inequalities.
\end{abstract}




\section{§ 1. Introduction}

It is well known (see [C, S]), that rarefaction shocks, i.e., shocks which violate the Lax entropy condition, in strictly hyperbolic, genuinely nonlinear systems (see $[\mathrm{S}]$ ) are unstable solutions of nonlinear hyperbolic systems of conservation laws. In this paper we clarify the precise sense in which such solutions are unstable for systems of equations.

We say that a shock wave is unstable in the class of smooth solutions if there exists a sequence of $C^{2}$-solutions, defined uniformly for $x \in \mathbb{R}, 0 \leqq t \leqq \bar{t}$ for some $\bar{t}>0$, that converges in every $L^{p}$-class $(p \geqq 1)$ to the given discontinuous data at $t=0$, but does not converge to the given shock-wave solution for any $t$, $0<t \leqq \bar{t}$. For scalar conservation laws, it is easy to show that rarefaction shocks are unstable in the class of smooth solutions. For systems of equations, the situation is more complicated, since, generally speaking, the condition that the family of smooth solutions converges to the discontinuous data at $t=0$ implies that such smooth solutions cannot be constructed uniformly on $\mathbb{R} \times[0, \bar{t}]$ for any $\bar{t}>0$. In this paper we shall show by means of elementary constructions that any shock wave solution of a general system of conservation laws is unstable in the class of smooth solutions, whenever there exists another solution to the Riemann problem having the same data that consists entirely of constant states separated only by rarefaction waves and contact discontinuities, where at least one of the rarefaction waves is of non-zero strength. (By a shock wave, we mean any solution consisting of two constant states separated by a jump discontinuity that satisfies the Rankine-Hugoniot jump relation (see [S]).) For $2 \times 2$ systems of strictly hyperbolic, genuinely nonlinear systems, we show that this condition is both necessary and sufficient. We also prove that for the full $3 \times 3$ Euler equations of gas dynamics having a polytropic equation of state, rarefaction shocks of "moderate" strength (see Theorem 8) are unstable in the class of smooth solutions if and only if the adiabatic gas constant $\gamma$ satisfies $1<\gamma<\frac{5}{3}$. Thus for this range of $\gamma$, the theory of the Riemann problem for Euler's equations can be rigorously developed (for moderate shocks, but we conjecture for all shocks), by using stability with respect to smoothing as a criterion for admissibility, instead of the classical entropy inequalities. We thus see that gas dynamics is special because for general systems, the instability of rarefaction shocks can only be seen within a class of solutions which is larger than the class of smooth solutions, and hence for such equations, an "entropy condition" must be provided in order to observe instability. We hope that this observation might clarify the idea of using stability as a criterion for admissibility in non-strictly hyperbolic systems where general entropy conditions are still unknown. The example of gas dynamics demonstrates that this can be done for important systems.

In the final section of this paper, we show how our study of rarefaction shocks leads to a seemingly paradoxical situation, whereby it appears that a Riemann problem admits two distinct admissible solutions. Our example demonstrates that some care must be taken when one solves Riemann problems in the $(u, p)$-plane, cf. [CF, S].

Throughout this paper we assume that the reader is familiar with the standard "shock-wave" notation and terminology as discussed in [S], for example. 


\section{§ 2. The Instability Theorem}

We consider $n \times n$ systems of nonlinear hyperbolic conservation laws in one space dimension

$$
u_{t}+f(u)_{x}=0
$$

where $u=\left(u_{1}, \ldots, u_{n}\right), x \in \mathbb{R}, t>0$ and $f \in C^{3}(\Omega)$, where $\Omega$ is a domain in $\mathbb{R}^{n}$. Except where noted, we assume that the system is strictly hyperbolic and either genuinely nonlinear or linearly degenerate in each characteristic field (see [S]). In this case, let $\lambda_{1}<\ldots<\lambda_{n}$ denote the eigenvalues of $d f$, (the first derivative of the vector function $f$ ), with corresponding eigenvectors $r_{1}, \ldots, r_{n}$. For $u_{L} \in \Omega$, let $\mathscr{R}_{i}\left(u_{L}\right)$ denote the integral curve of the $i^{\text {th }}$ eigenvector field through $u_{L}$ and let $\mathscr{S}_{i}\left(u_{L}\right)$ denote the $i^{\text {th }}$ Hugoniot locus of $u_{L}$ (i.e., $u_{R} \in \mathscr{S}_{i}\left(u_{L}\right) \cap \Omega$ corresponds to a discontinuity, say of speed $s$, determined by the $i^{\text {th }}$ characteristic field). We consider piecewise constant initial data

$$
u_{0}(x)= \begin{cases}u_{L}, & x \leqq 0 \\ u_{R}, & x>0\end{cases}
$$

The first result applies to an arbitrary system of conservation laws of the form (1). Let $u(x, t)$ denote a (weak) solution of the Riemann problem (1), (2), which is a shock of speed $s$, and let $\left[u_{L}, u_{R}\right](x, t)$ denote a second solution of this same Riemann problem.

Theorem 1. If there exists a second solution $\left[u_{L}, u_{R}\right]$ which consists of $m$ waves, each of which is either a rarefaction wave or a contact discontinuity, and if at least one of the rarefaction waves is nonzero, then there exists a one-parameter family $u_{\varepsilon}(x, t)$ of $C^{2}$-solutions of (1) satisfying

$$
\begin{array}{r}
\lim _{\varepsilon \rightarrow 0}\left\|u_{\varepsilon}(\cdot, 0)-u_{0}(\cdot)\right\|_{L^{p}}=0, \\
\lim _{\varepsilon \rightarrow 0}\left\|u_{\varepsilon}(\cdot t)-u(\cdot, t)\right\|_{L^{p}}>0
\end{array}
$$

for all $p>0$ and all $t>0$.

Proof. Assume that $\left[u_{L}, u_{R}\right](x, t)$ is a solution of (1), (2) consisting of $m$ waves, each of which is either a rarefaction wave or a contact discontinuity. Then there exist states $u_{L}=u^{0}, u^{1}, \ldots, u^{m}=u_{R}$ such that $u^{i} \in \mathscr{R}_{i}\left(u^{i-1}\right)$ and such that the $i^{\text {th }}$ waves take $u^{i-1}$ to $u^{i}$. Let $\mathscr{R}_{i}(\sigma), 0 \leqq \sigma \leqq 1$ denote a smooth parametrization of $\mathscr{R}_{i}\left(u^{i-1}\right)$ between $u^{i-1}$ and $u^{i}$ satisfying $\mathscr{R}_{i}(0)=u^{i-1}, \mathscr{R}_{i}(1)=u^{i}$, $i=1, \ldots, m$. Let $\sigma(x)$ denote an increasing $C^{\infty}$-function, $\sigma:[0, \varepsilon] \rightarrow[0,1]$, satisfying

$$
\sigma(x)= \begin{cases}0, & x<0, \\ 1, & x \geqq \varepsilon .\end{cases}
$$

For each $\varepsilon>0$, define initial data by

$$
u_{\varepsilon}^{i}(x, 0)=\mathscr{R}_{i}(\sigma(x-(i-1) \varepsilon)) .
$$


The solution $u_{c}^{i}(x, t)$ of (1), (4) is a $C^{2}$-function consisting of an $i$-simple wave in which a state $u=\mathscr{R}_{i}(\sigma)$ propagates with speed

Thus from (3),

$$
d x / d t=\lambda_{i}\left(\mathscr{R}_{i}(\sigma)\right), \quad 0<\sigma<1 .
$$

$$
u_{\varepsilon}^{i}(x, t)= \begin{cases}u^{i-1}, & x<\lambda_{i}\left(u^{i-1}\right) t+(i-1) \varepsilon, \\ u^{i}, & x \geqq \lambda_{i}\left(u^{i}\right) t+(i-1) \varepsilon+\varepsilon=\lambda_{i}\left(u^{i}\right) t+i \varepsilon .\end{cases}
$$

Therefore in $t>0$, we can construct a $C^{2}$-solution $u_{\varepsilon}(x, t)$ consisting of $m$ noninteracting simple waves by taking ( $c f$. Figure 1 ),

$$
u_{\varepsilon}(x, t)= \begin{cases}u_{\varepsilon}^{1}(x, t), & \text { if } x \leqq \lambda_{1}\left(u^{1}\right) t+\varepsilon, \\ u_{\varepsilon}^{i}(x, t), & \text { if } \lambda_{i-1}\left(u^{i-1}\right) t+(i-1) \varepsilon \leqq x<\lambda_{i}\left(u^{i}\right) t+i \varepsilon, \\ u_{\varepsilon}^{m}(x, t), & \text { if } \lambda_{m}\left(u^{m}\right) t+m \varepsilon \leqq x\end{cases}
$$

Note that $u_{\varepsilon}$ is a $C^{2}$-function since $f$ is $C^{3}$.

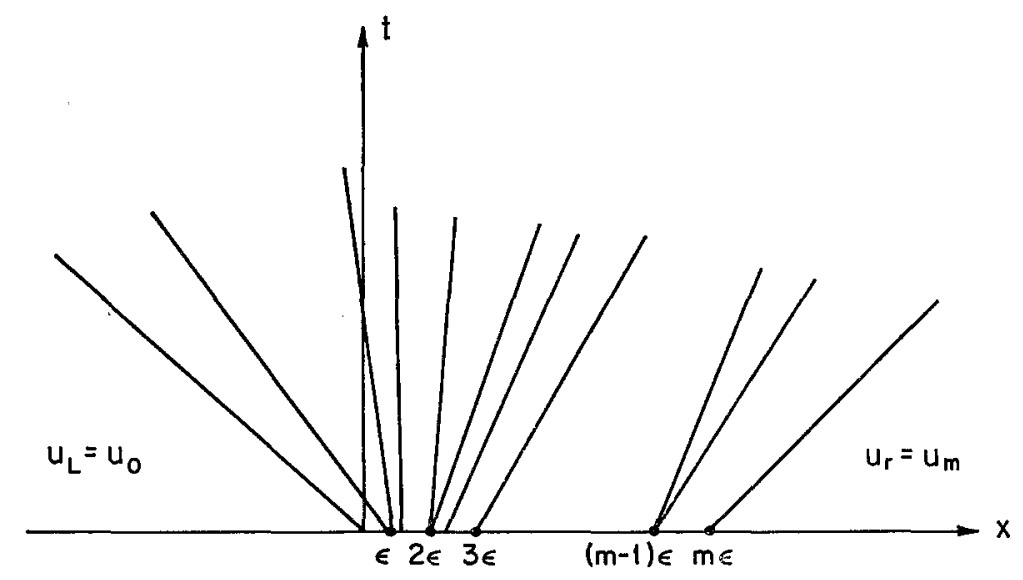

Fig. 1

It is clear that

$$
u_{\varepsilon}(x, t)= \begin{cases}u_{L}, & \text { if } x \leqq \lambda_{1}\left(u_{L}\right) t \\ u_{R}, & \text { if } x>\lambda_{m}\left(u_{R}\right) t+m \varepsilon\end{cases}
$$

so that for any $p>0$,

$$
\lim _{\varepsilon \rightarrow 0}\left\|u_{\varepsilon}(\cdot, 0)-u_{0}(\cdot)\right\|_{L^{p}}=0 .
$$

Furthermore, since $u_{\varepsilon}^{i}$ tends to the rarefaction wave or the contact discontinuity connecting $u^{i-1}$ to $u^{i}$ and since at least one of the rarefaction waves is expansive, it follows at once that for any $t>0$,

$$
\lim _{\varepsilon \rightarrow 0}\left\|u_{\varepsilon}(\cdot, t)-u(\cdot, t)\right\|_{L^{p}}>0 .
$$

This completes the proof of the theorem. 
We shall now derive a consequence of our result. First, note that in the case of the $2 \times 2$ Johnson-Smoller systems [JS], which include the equations of isentropic gas dynamics, the interaction of two shocks of the same family produces a shock of that family, together with a rarefaction wave of the opposite family. This implies that for both characteristic families, the rarefaction shock curves $\mathscr{B}_{i}\left(u_{L}\right), i=1$ or 2 , break into the concave side of the integral curve through $u_{L}$, and as was shown in [CS], they do not leave that region. Hence, if $u_{R} \in \mathscr{B}_{i}\left(u_{L}\right)$, then the solution of the Riemann problem (1), (2) consists of two rarefaction waves (see Figure 2). Thus Theorem 1 implies the following result which was first proved in [CS].

Corollary 2. For the $2 \times 2$ Johnson-Smoller systems, (see [JS]), rarefaction shocks are unstable in the class of smooth solutions.

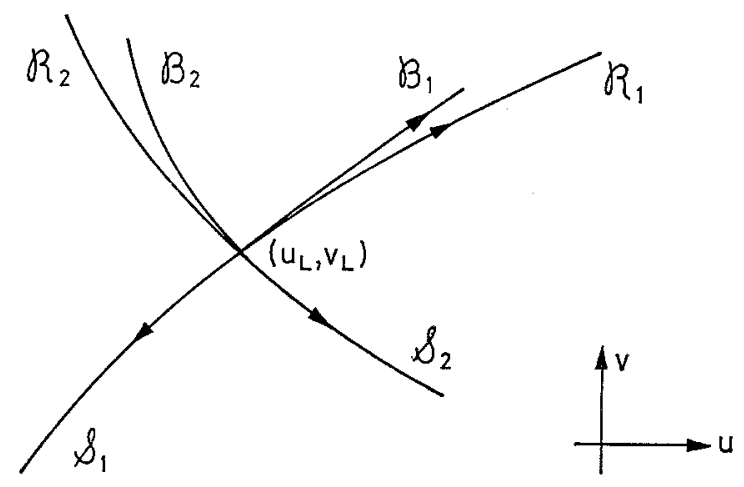

Fig. 2

\section{§ 3. Applications to Gas Dynamics}

We consider in this section the full $3 \times 3$ gas dynamics (Euler) equations with an ideal, polytropic equation of state [S]; we shall show that Theorem 1 applies to shocks of "moderate" strength, provided that $1<\gamma<\frac{5}{3}$, where $\gamma$ denotes the adiabatic gas constant. The meaning of the term "moderate" will be made precise below (see Theorem 8). Our technique is to study the projections of the shock and rarefaction wave curves in the $(u, p)$-plane (see [CF, S]). There we shall show that only for $1<\gamma<\frac{5}{3}$ do the rarefaction shock curves break into the "quadrant" determined by the backward and forward rarefaction wave curves (see Figure 3). But somewhat surprisingly, these curves must eventually leave this quadrant. Thus for weak rarefaction shocks, Theorem 1 applies and shows that they are unstable in the class of smooth solutions if and only if $1<\gamma<\frac{5}{3}$. On the other hand, we can (uniformly) estimate when these curves leave the "rarefaction wave quadrant", and this enables us to assert the same instability result for rarefaction shocks of "moderate" strength. We proceed with the details. 

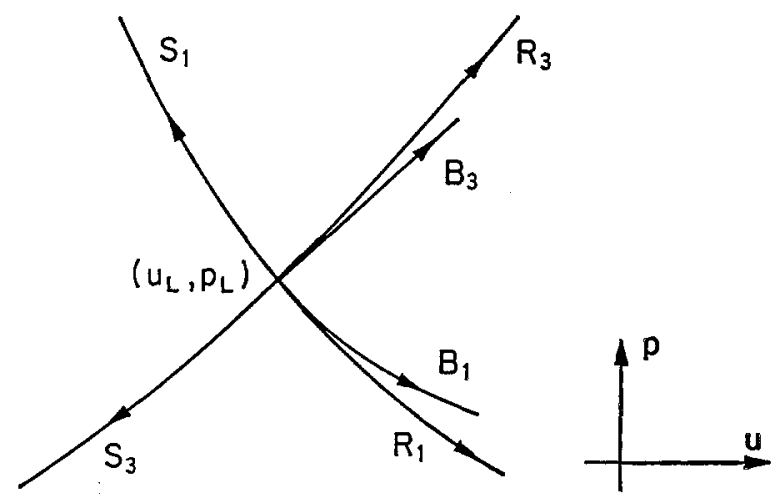

Fig. 3

In the $(u, p)$-plane, for ideal polytropic gases, the shock and rarefaction wave curves starting at the state $\left(u_{L}, p_{L}, \varrho_{L}\right)$ on the left have the following form (see $[\mathrm{S}, \mathrm{Ch} .18, \S \mathrm{C}])$ :

$\begin{array}{ll}S_{1}\left(u_{L}, p_{L}, \varrho_{L}\right): \text { 1-shock wave: } & u-u_{L}=-\phi_{L}(p), \quad u<u_{L}, \quad p>p_{L}, \\ S_{3}\left(u_{L}, p_{L}, \varrho_{L}\right): 3 \text {-shock wave: } & u-u_{L}=\phi_{L}(p), \quad u<u_{L}, \quad p_{L}>p,\end{array}$

$R_{1}\left(u_{L}, p_{L}, Q_{L}\right):$ 1-rarefaction wave: $u-u_{L}=-\psi_{L}(p), \quad u>u_{L}, \quad p_{L}>p$, $R_{3}\left(u_{L}, p_{L}, \varrho_{L}\right): 3$-rarefaction wave: $u-u_{L}=\psi_{L}(p), \quad u>u_{L}, \quad p>p_{L}$ where $(u, p, \varrho)$ is connected to $\left(u_{L}, p_{L}, \varrho_{L}\right)$ on the right. Here

$$
\begin{aligned}
& \phi_{L}(p)=\left(p-p_{L}\right) \sqrt{\frac{\left(1-\mu^{2}\right) v_{L}}{p+\mu^{2} p_{L}}} \\
& \psi_{L}(p)=\frac{\sqrt{1-\mu^{4}}}{\mu^{2}} \sqrt{v_{L}} p_{L}^{\frac{1}{2 \gamma}}\left(p^{\frac{\gamma-1}{2 \gamma}}-p_{L}^{\frac{\gamma-1}{2 \gamma}}\right),
\end{aligned}
$$

and $\mu^{2}=(\gamma-1)(\gamma+1)^{-1}, v=\varrho^{-1}$, where $\varrho$ is the density, $p$ is the pressure, and $u$ is the velocity. The rarefaction shock curves take the form

$$
\begin{aligned}
& B_{1} \text { : 1-rarefaction shocks: } u-u_{L}=-\phi_{L}(p), \quad u>u_{L}, \quad p_{L}>p, \\
& B_{3} \text { : 3-rarefaction-shocks: } u-u_{L}=\phi_{L}(p), u>u_{L}, \quad p>p_{L} \text {. }
\end{aligned}
$$

In order to prove these assertions, we must first show that the curves $B_{1}$ and $B_{3}$ break into the $R_{1}-R_{3}$ region.

Lemma 3. For $(u, p) \in B_{1} \cup B_{3}$ and $\left|u-u_{L}\right|+\left|p-p_{L}\right|$ sufficiently small, $(u, p)$ lies in the $R_{1}-R_{3}$ region if and only if $1<\gamma<\frac{5}{3}$. If $\gamma=\frac{5}{3}$ and $(u, p) \in B_{3}$, then $(u, p)$ lies in the $R_{1}-R_{3}$ region, while if $(u, p) \in B_{1}$, then $(u, p)$ does not lie in this region. 
Proof. We compute

$$
\begin{gathered}
\phi_{L}^{\prime}(p)=\frac{p+\left(2 \mu^{2}+1\right) p_{L}}{2\left(p+\mu^{2} p_{L}\right)^{3 / 2}}\left[\left(1-\mu^{2}\right) v_{L}\right]^{1 / 2}>0 \\
\phi_{L}^{\prime \prime}(p)=-\frac{p+\left(4 \mu^{2}+3\right) p_{L}}{4\left(p+\mu^{2} p_{L}\right)}\left[\left(1-\mu^{2}\right) v_{L}\right]^{1 / 2} \\
\phi_{L}^{\prime \prime \prime}(p)=\frac{3}{8} \frac{p+\left(6 \mu^{2}+5\right) p_{L}}{\left(p+\mu^{2} p_{L}\right)}\left[\left(1-\mu^{2}\right) v_{L}\right]^{1 / 2}, \\
\psi_{L}^{\prime}(p)=\frac{\gamma-1}{2 \gamma} p^{-\left(\frac{1-\gamma}{2 \gamma}\right) \frac{\left[\left(1-\mu^{4}\right) v_{L}\right]^{1 / 2}}{\mu^{2}} p_{L}^{\frac{1}{2 \gamma}},} \\
\psi_{L}^{\prime \prime}(p)=-\frac{\gamma-1}{2 \gamma}\left(\frac{1+\gamma}{2 \gamma}\right) p^{-\left(\frac{1+3 \gamma}{2 \gamma}\right)} \frac{\left[\left(1-\mu^{4}\right) v_{L}\right]^{1 / 2}}{\mu^{2}} p_{L}^{\frac{1}{2 \gamma}}, \\
\psi_{L}^{\prime \prime \prime}(p)=\frac{\gamma-1}{2 \gamma}\left(\frac{1+\gamma}{2 \gamma}\right)\left(\frac{1+3 \gamma}{2 \gamma}\right) p^{-\left(\frac{1+5 \gamma}{2 \gamma}\right)} \frac{\left[\left(1-\mu^{4}\right) v_{L}\right]^{1 / 2}}{\mu^{2}} p_{L}^{\frac{1}{2 \gamma}} .
\end{gathered}
$$

From these expressions, one easily checks that

$$
\begin{aligned}
& \phi_{L}^{\prime}\left(p_{L}\right)=\psi_{L}^{\prime}\left(p_{L}\right), \quad \phi_{L}^{\prime}\left(p_{L}\right)=\psi_{L}^{\prime \prime}\left(p_{L}\right), \\
& \phi_{L}^{\prime \prime \prime}\left(p_{L}\right)-\psi_{L}^{\prime \prime \prime}\left(p_{L}\right)= \frac{9}{4} \frac{\left[\left(1-\mu^{2}\right) v_{L}\right]^{1 / 2}}{\left[\left(\mu^{2}+1\right) p_{L}\right]^{5 / 2}}-\frac{\left(\gamma^{2}-1\right)(1+3 \gamma)}{8 \gamma^{3}} p_{L}^{-5 / 2} \frac{\left[\left(1-\mu^{4}\right) v_{L}\right]^{1 / 2}}{\mu^{2}} \\
&= \frac{1}{8} \frac{\left[\left(1-\mu^{2}\right) v_{L}\right]^{1 / 2}}{\left[\left(\mu^{2}+1\right) p_{L}\right]^{5 / 2}}\left[18-\frac{\left(\mu^{2}+1\right)^{3}\left(\gamma^{2}-1\right)(1+3 \gamma)}{\gamma^{3} \mu^{2}}\right] \\
&=\frac{1}{8} \frac{\left[\left(1-\mu^{2}\right) v_{L}\right]^{1 / 2}}{\left[\left(\mu^{2}+1\right) p_{L}\right]^{5 / 2}} \frac{2}{\gamma-1}(5-3 \gamma) .
\end{aligned}
$$

Thus $\phi_{L}^{\prime \prime \prime}\left(p_{L}\right)-\psi_{L}^{\prime \prime \prime}\left(p_{L}\right)>0$ if $1<\gamma<\frac{5}{3}$, and $\phi_{L}^{\prime \prime \prime}\left(p_{L}\right)-\psi^{\prime \prime \prime}\left(p_{L}\right)<0$ if $\gamma>\frac{5}{3}$. Now let $p<p_{L}$, with $p$ near $p_{L}$, and let $\left(u_{1}, p\right) \in B_{1}, \quad\left(u_{2}, p\right) \in R_{1}$; then

$$
\begin{aligned}
u_{2}-u_{1} & =\left(u_{2}-u_{L}\right)-\left(u_{1}-u_{L}\right)=-\psi_{L}(p)-\left(-\phi_{L}(p)\right)=\phi_{L}(p)-\psi_{L}(p) \\
& =\frac{\left(p-p_{L}\right)^{3}}{3 !}\left(\phi_{L}^{\prime \prime \prime}\left(p_{L}\right)-\psi_{L}^{\prime \prime \prime}\left(p_{L}\right)\right)+O_{4}\left(\left|p-p_{L}\right|\right)
\end{aligned}
$$

so that $u_{2}<u_{1}$ if $1<\gamma<\frac{5}{3}$, and $u_{2}>u_{1}$ if $\gamma>\frac{5}{3}$. Thus the $B_{1}$ curve breaks into the $R_{1}-R_{3}$ region if $1<\gamma<\frac{5}{3}$, and breaks out of this region if $\gamma>\frac{5}{3}$. Similarly, we can check that if $\gamma>\frac{5}{3}$, the $B_{3}$ curve breaks out of the $R_{1}-R_{3}$ region, while if $1<\gamma<\frac{5}{3}$, the $B_{2}$ curve breaks into this region. Moreover, a calculation gives

$$
\begin{aligned}
\phi_{L}^{\prime \prime \prime \prime}(p) & =-\frac{15}{16} \frac{p+\left(8 \mu^{2}+7\right) p_{L}}{\left[p+\mu^{2} p_{L}\right]^{9 / 2}}\left[\left(1-\mu^{2}\right) v_{L}\right]^{1 / 2}, \\
\psi_{L}^{\prime \prime \prime \prime}(p) & =\frac{\left(1+\gamma^{2}\right)(1+3 \gamma)(1+5 \gamma)}{16 \gamma^{4}} p^{-\left(\frac{1+7 \gamma}{2 \gamma}\right) \frac{\left[\left(1-\mu^{4}\right) v_{L}\right]^{1 / 2}}{\mu^{2}} p_{L}^{\frac{1}{2 \gamma}}}
\end{aligned}
$$


so that

$$
\begin{aligned}
\phi_{L}^{\prime \prime \prime \prime}\left(p_{L}\right)-\psi_{L}^{\prime \prime \prime \prime} & \left(p_{L}\right) \\
& =\frac{\left[\left(1-\mu^{2}\right) v_{L}\right]^{1 / 2}}{\left[\left(\mu^{2}+1\right) p_{L}\right]^{7 / 2}}\left[-\frac{15}{2}+\frac{\left(1+\mu^{2}\right)^{4}}{\mu^{2}} \frac{\left(\gamma^{2}-1\right)}{16 \gamma^{4}}(1+3 \gamma)(1+5 \gamma)\right] \\
& =\frac{1}{2} \frac{\left[\left(1-\mu^{2}\right) v_{L}\right]^{1 / 2}}{\left[\left(\mu^{2}+1\right) p_{L}\right]^{7 / 2}}\left(15 \gamma^{2}-14 \gamma-13\right) .
\end{aligned}
$$

But at $\gamma=\frac{5}{3}, 15 \gamma^{2}-14 \gamma-13=\frac{1}{3}(125-70-39)>0$. Thus, if $\left(u_{1}, p\right) \in B_{1}$, $\left(u_{2}, p\right) \in R_{1}$, and $p$ is near $p_{L}$, we have

$$
\begin{aligned}
u_{2}-u_{1} & =\left(u_{2}-u_{L}\right)-\left(u_{1}-u_{L}\right)=-\psi_{L}(p)-\left(-\phi_{L}(p)\right) \\
& =\phi_{L}(p)-\psi_{L}(p)=\frac{\left(p-p_{L}\right)^{4}}{4 !}\left(\phi_{L}^{\prime \prime \prime \prime}\left(p_{L}\right)-\psi^{\prime \prime \prime \prime}\left(p_{L}\right)\right)+O_{5} \\
& >0
\end{aligned}
$$

so that the $B_{1}$ curve breaks out of the $R_{1}-R_{3}$ region if $\gamma=\frac{5}{3}$. On the other hand if $\left(u_{1}, p\right) \in B_{3}$ and $\left(u_{2}, p\right) \in R_{3}$, then at $\gamma=\frac{5}{3}, u_{2}-u_{1}=\psi_{2}(p)-\phi_{2}(p)$ $<0$, so that the $B_{3}$ curve breaks into the $R_{1}-R_{3}$ region. This completes the proof.

Remark. The apparent lack of symmetry between $B_{1}$ and $B_{3}$ implied by the last result is only illusory. In fact the symmetry is regained if one starts with states $U_{r}$; in this context, the roles of $B_{1}$ and $B_{3}$ are reversed.

The next lemma shows that the rarefaction-shock curves must eventually leave the $R_{1}-R_{3}$ region. In order to state the precise result, we consider a given state $\left(u_{r}, p_{r}, \varrho_{r}\right)$ on the right, and denote by $\tilde{R}_{i}, \tilde{S}_{i}, \tilde{B}_{i},(i=1$, or 3$)$, the corresponding rarefaction waves, shock waves and rarefaction shocks, respectively, which can be connected to $\left(u_{r}, p_{r}, \varrho_{r}\right)$ on the left by the given wave (cf. Figure 4). More precisely, we have

$$
\begin{array}{r}
\tilde{S}_{1}\left(u_{r}, p_{r}, \varrho_{r}\right): \text { 1-shock wave: } u-u_{r}=-\phi_{r}(p), p_{r}>p, u>u_{r}, \\
\tilde{S}_{3}\left(u_{r}, p_{r}, \varrho_{r}\right): \text { 3-shock wave: } u-u_{r}=\phi_{r}(p), \quad p>p_{r}, u>u_{r}, \\
\tilde{R}_{1}\left(u_{r}, p_{r}, \varrho_{r}\right): \text { 1-rarefaction wave: } u-u_{r}=-\psi_{r}(p), \quad p>p_{r}, u_{r}>u, \\
\tilde{R}_{3}\left(u_{r}, p_{r}, \varrho_{r}\right): \text { 3-rarefaction wave: } u-u_{r}=\psi_{r}(p), p_{r}>p, u>u_{r}, \\
\tilde{B}_{1}\left(u_{r}, p_{r}, \varrho_{r}\right): \text { 1-rarefaction-shock: } u-u_{r}=-\phi_{r}(p), p_{r}>p, u_{r}>u, \\
\tilde{B}_{3}\left(u_{r}, p_{r}, \varrho_{r}\right): \text { 3-rarefaction-shock: } u-u_{r}=\psi_{r}(p), p_{r}>p, u_{r}>u .
\end{array}
$$

Here the functions $\phi_{r}(p)$ and $\psi_{r}(p)$ are defined by (7) and (8), respectively, where we replace the subscript $L$ by $r$.

The following result shows that the statement in the last lemma does not hold in the large; $c f .(6),(9),(10),(11)$. 


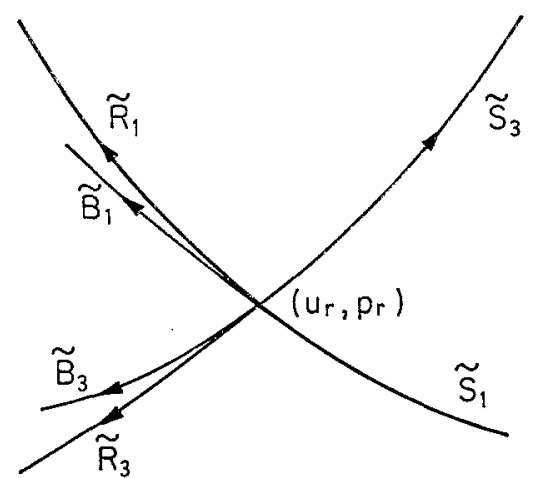

Fig. 4

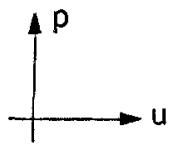

Lemma 4. Let $1<\gamma<\frac{5}{3}$; then

(a) The $B_{1}$ curve, $\left(u-u_{L}=-\phi_{L}(p), \quad p_{L}>p, u>u_{L}\right)$ must eventually leave the $R_{1}-R_{3}$ region. More precisely, there is a $\bar{p}<p_{L}$ such that if $p<\bar{p}$, any point $(u, p) \in B_{1}$ lies in the $R_{1}-S_{3}$ quadrant of $\left(u_{L}, p_{L}\right) ;$ cf. Figure 3. (b) The $\tilde{B_{3}}$ curve, $\left(u-u_{r}=\phi_{r}(p), p_{r}>p, u_{r}>u\right)$ must eventually leave the $\tilde{R}_{1}-\tilde{R}_{3}$ region. More precisely, there is a $\bar{p}<p_{r}$ such that if $p<\bar{p}$, any point $(u, p) \in \tilde{B}_{3}$ lies in the $\widetilde{S}_{1}-\widetilde{R}_{3}$ quadrant of $\left(u_{r}, p_{r}\right)$; cf. Figure 4.

Proof. We only prove (a) since the proof of (b) is similar. For this we show thah the $B_{1}$ curve, starting at $\left(u_{L}, p_{L}\right)$, crosses the $R_{1}$ curve, starting at $\left(u_{L}, p_{L}\right)$.

Thus from (6)-(9), we have

$$
\begin{aligned}
& B_{1}: u-u_{L}=\phi_{L}(p)=\left(p_{L}-p\right) \sqrt{\frac{\left(1-\mu^{2}\right) v_{L}}{p+\mu^{2} p_{L}}}, \quad p<p_{L}, \\
& R_{1}: u-u_{L}=-\psi_{L}(p)=\frac{\sqrt{1-\mu^{4}}}{\mu^{2}} \sqrt{v_{L}} \frac{1}{p_{L}^{2 \gamma}}\left(p^{\frac{\gamma-1}{2 \gamma}}-\frac{\gamma-1}{p_{L}^{2 \gamma}}\right), \quad p<p_{L} .
\end{aligned}
$$

Also, for $p$ near $p_{L}$, Lemma 3 implies that

Furthermore,

$$
\phi_{L}(p)>\psi_{L}(p)
$$

$$
\begin{aligned}
\phi_{L}(0)-\psi_{L}(0) & =\frac{\sqrt{1-\mu^{2}}}{\mu}\left(p_{L} v_{L}\right)^{1 / 2}-\sqrt{\frac{1-\mu^{4}}{\mu^{4}}} \sqrt{v_{L} p_{L}} \\
& =\sqrt{\frac{2 p_{L} v_{L}}{\gamma-1}}\left(1-\sqrt{\frac{2 \gamma}{\gamma-1}}\right)<0,
\end{aligned}
$$

as $\gamma>1$. This together with (12) gives our result.

The next lemma shows that the opposite is true for the $B_{3}$ and $\tilde{B}_{1}$ curves ( $c f$. the remark following the proof of Lemma 3). 
Lemma 5. Let $1<\gamma \leqq \frac{5}{3}$.

(a) The $B_{3}$ curve $\left(u-u_{L}=\phi_{L}(p), u>u_{L}, p>p_{L}\right)$ never leaves the $R_{1}-R_{3}$ region.

(b) The $\tilde{B_{1}}$ curve $\left(u-u_{r}=-\phi_{r}(p), p>p_{r}, u_{r}>u\right)$ never leaves the $\tilde{R}_{1}-\tilde{R}_{3}$ region.

Proof. Again we shall only prove (a). In order to do this, we use the parametrization of the $R_{3}$ and $B_{3}$ curves given in [S, p. 354]. Thus the $u$-components of these curves are given by

$$
\begin{array}{ll}
R_{3}: \frac{\gamma-1}{2 c_{L}}\left(u-u_{L}\right)=e^{\tau x}-1 \equiv h(x), & x \geqq 0, \\
B_{3}: \frac{\gamma-1}{2 c_{L}}\left(u-u_{L}\right)=\sqrt{\tau} \frac{e^{x}-1}{\sqrt{1+\beta e^{x}}} \equiv g(x), & x \geqq 0,
\end{array}
$$

where $\tau=(\gamma-1) / 2 \gamma$ and $\beta=(\gamma+1) /(\gamma-1)$. Let

We have

$$
f(x)=h(x)-g(x), \quad x \geqq 0 \text {. }
$$

$$
f(0)=0, \quad f(x)>0 \quad \text { for } x \text { near } 0, x>0 .
$$

We shall show that $f(x)>0$ for all $x>0$. To do this it suffices to show:

$$
\text { if } x>0 \text { and } f(x)=0 \text {, then } f^{\prime}(x)>0 \text {. }
$$

Thus at such a point, we compute

$$
\begin{aligned}
f^{\prime}(x) & =\frac{\sqrt{\tau}}{2} \frac{1}{\sqrt{1+\beta e^{x}}}\left[2 e^{x}-\frac{e^{x}-1}{1+\beta e^{x}} \beta e^{x}-2 \sqrt{\tau\left(1+\beta e^{x}\right)}-2 \tau\left(e^{x}-1\right)\right] \\
& =\frac{\sqrt{\tau}}{2} \frac{1}{\sqrt{1+\beta e^{x}}}\left[(1+2 \tau)+(1-2 \tau) e^{x}+\frac{e^{x}-1}{1+\beta e^{x}}-2 \sqrt{\tau\left(1+\beta e^{x}\right)}\right] .
\end{aligned}
$$

If we write

$$
\phi(x)=\left[(1+2 \tau)+(1-2 \tau) e^{x}+\frac{e^{x}-1}{1+\beta e^{x}}-2 \sqrt{\tau\left(1+\beta e^{x}\right)}\right],
$$

then to prove (15) it is enough to show that

Now $\phi(0)=0$ and

$$
\phi(x)>0 \quad \text { if } x>0 .
$$

$$
\phi^{\prime}(x)=e^{x}\left[(1-2 \tau)+\frac{1}{1+\beta e^{x}}+\frac{\beta\left(1-e^{x}\right)}{\left(1+\beta e^{x}\right)^{2}}-\frac{\beta \tau}{\sqrt{\tau\left(1+\beta e^{x}\right)}}\right] .
$$

If we set $\psi(x)=\phi^{\prime}(x) e^{-x}$, then it suffices to show that

$$
\psi(x)>0 \quad \text { if } x>0 \text {. }
$$


Now as

$$
\frac{1}{1+\beta e^{x}}+\frac{\beta\left(1-e^{x}\right)}{\left(1+\beta e^{x}\right)^{2}}=\frac{1+\beta}{\left(1+\beta e^{x}\right)^{2}} \quad \text { and } \quad(1-2 \tau)=1 / \gamma
$$

we may write

$$
\psi(x)=\frac{1}{\gamma}+\frac{(1+\beta)}{\left(1+\beta e^{x}\right)^{2}}-\frac{\beta \sqrt{\tau}}{\sqrt{1+\beta e^{x}}} .
$$

Thus (19) will hold if

$\frac{1}{\gamma^{2}}+\frac{2}{\gamma}(1+\beta) \frac{1}{\left(1+\beta e^{x}\right)^{2}}+\frac{(1+\beta)^{2}}{\left(1+\beta e^{x}\right)^{4}}-\frac{\beta^{2} \tau}{\left(1+\beta e^{x}\right)}>0 \quad$ for $x>0$.

The left-hand side of (20) can be rewritten as

$$
\frac{\left(1+\beta e^{x}\right)^{4}+2 \gamma(1+\beta)\left(1+\beta e^{x}\right)^{2}+(1+\beta)^{2} \gamma^{2}-\beta^{2} \tau \gamma^{2}\left(1+\beta e^{x}\right)^{3}}{\gamma^{2}\left(1+\beta e^{x}\right)^{4}} .
$$

Set

$$
y=1+\beta e^{x} \quad(y \geq 1+\beta) ;
$$

then the numerator in (21) takes the form

$$
A(y)=y^{4}-\beta^{2} \tau \gamma^{2} y^{3}+2 \gamma(1+\beta)^{2} y^{2}+(1+\beta)^{2} \gamma^{2} .
$$

Now $A(0)>0, A(y) \rightarrow \infty$ as $y \rightarrow \infty$, and $A$ has at most two positive roots. But using the relations

$$
\beta^{2} \tau \gamma^{2}=\frac{\gamma(\gamma+1)^{2}}{2(\gamma-1)}, \quad 2 \gamma(1+\beta)=\frac{4 \gamma^{3}}{\gamma-1}, \quad \gamma^{2}(1+\beta)^{2}=\frac{4 \gamma^{4}}{(\gamma-1)^{2}},
$$

one easily checks that $A(1+\beta)=0$ and

$$
\begin{aligned}
A^{\prime}(1+\beta) & =A^{\prime}(2 \gamma /(1+\gamma)) \\
& =\frac{2 \gamma^{3}}{(\gamma-1)^{3}}[5+\gamma(2-3 \gamma)] .
\end{aligned}
$$

Thus if $\gamma<\frac{5}{3}$, then $A^{\prime}(1+\beta)>0$, while $A^{\prime}(1+\beta)=0$ if $\gamma=\frac{5}{3}$. It follows that $A$ has exactly two positive roots, with $1+\beta$ being the larger one. Thus $A(y)>0$ if $y>1+\beta$, so from (22) we see that (20) holds and the proof of Lemma 5 is complete.

In view of the last two lemmas, we see that for $1<\gamma<\frac{5}{3}$, the geometry in the $(u, p)$-plane can be depicted as in Figure 5.

We now estimate the strength of the shocks corresponding to points on $B_{1}$ (or $\tilde{B}_{3}$ ) inside the $R_{1}-R_{3}$ (or $\tilde{R}_{3}-\tilde{R}_{1}$ ) region. Note that Lemma 4 assures us that the curve $B_{1}$ (or $\tilde{B}_{3}$ ) crosses $R_{1}$ (or $\tilde{R}_{3}$ ) in an odd number of points in the region $p<p_{L}$ (or $p<p_{r}$ ). We next show that this crossing number is always equal to 1 . To this end, we again consider the parametrization of the $T_{1}$ and $B_{1}$ curves given in $[\mathrm{S} ; \mathrm{p}$. 354]. The $u$-components of these curves at their points of 


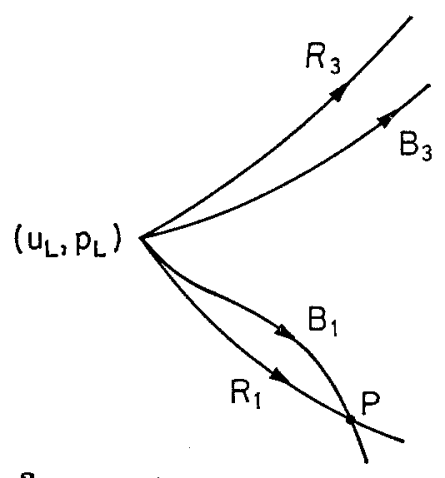

a

Fig. 5

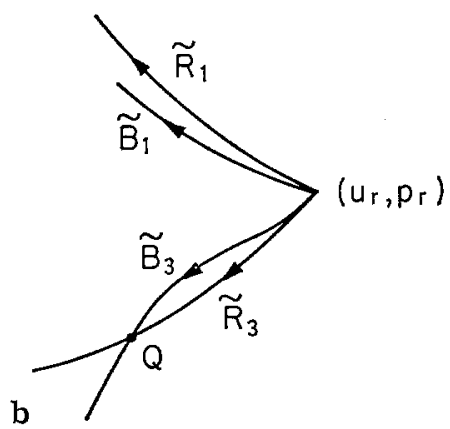

intersection $(\bar{u}, \bar{p})$ must satisfy the equation

$$
\frac{\sqrt{\tau}\left(1-e^{-x}\right)}{\left(1+\beta e^{-x}\right)^{1 / 2}}=1-e^{-\tau x}, \quad x \geqq 0, \quad e^{-x}=\bar{p} / p_{L} .
$$

If we set $y=e^{-x}$, then we are to find all roots of the equation

$$
\sqrt{\tau}(1-y)=\left(1-y^{\tau}\right)(1+\beta y)^{1 / 2}, \quad 0<y \leqq 1 .
$$

Clearly $y=1$ is a root. We now prove

Lemma 6. Let $1<\gamma<\frac{5}{3}$; then the equation (24) has exactly one root in $0<y<1$. If $\gamma=\frac{5}{3}$, (24) has no root in $0 \leqq y<1$; cf. Figure 6 .

Proof. Define $\phi(y)$ and $\psi(y)$ by

$$
\phi(y)=\left(1-y^{z}\right) \sqrt{1+\beta y}, \quad \psi(y)=\sqrt{\tau}(1-y), \quad 0 \leqq y \leqq 1 ;
$$

then (23) can be written as $\psi(y)=\phi(y)$. Notice that since $1<\gamma \leqq \frac{5}{3}$, it follows that $\tau=(\gamma-1) / 2 \gamma \leqq \frac{1}{5}$, so that $\psi(0)>\phi(0)$; moreover, $\phi(1)=\psi(1)$. We compute

where

$$
\begin{aligned}
\phi^{\prime}(y) & =-\tau y^{\tau-1} \sqrt{1+\beta y}+\frac{\beta}{2} \frac{\left(1-y^{\tau}\right)}{\sqrt{1+\beta y}}, \\
\phi^{\prime \prime}(y) & =\frac{y^{\tau-2}}{4(1+\beta y)^{3 / 2}} Q(y),
\end{aligned}
$$

Thus

$$
Q(y)=4 \tau(1+\beta y)[1-\tau(1+\beta y)]-\beta^{2} y^{2-\tau}+\beta^{2} y^{2} .
$$

$$
Q^{\prime}(y)=4 \tau \beta[1-2 \tau(1+\beta y)]-(2-\tau) \beta^{2} y^{1-\tau}+2 \beta^{2} y .
$$

An easy computation gives (since $\beta \geqq 4$ )

$$
\begin{aligned}
& Q(0)=4 \tau(1-\tau)>0, \quad Q^{\prime}(0)=4 \tau \beta(1-2 \tau)>0, \\
& Q(1)=0, \quad Q^{\prime}(1)=-4 \tau \beta+\tau \beta^{2}>0 \quad \text { if } \beta>4 \quad\left(1<y<\frac{5}{3}\right), \\
& Q^{\prime}(1)=0 \quad \text { if } \beta=4 \quad\left(\gamma=\frac{5}{3}\right) .
\end{aligned}
$$


Note also that

$$
\begin{gathered}
\phi^{\prime}(1)=\psi^{\prime}(1)=-\sqrt{\tau}, \quad \psi^{\prime \prime}(0)=\phi^{\prime \prime}(0)=0, \\
\psi^{\prime \prime \prime}(1)=\frac{Q^{\prime}(1)}{4(1+\beta)^{3 / 2}}>0=\phi^{\prime \prime \prime}(1) .
\end{gathered}
$$

Thus $\psi(y)$ is the tangent line of $\phi(y)$ at $y=1$.

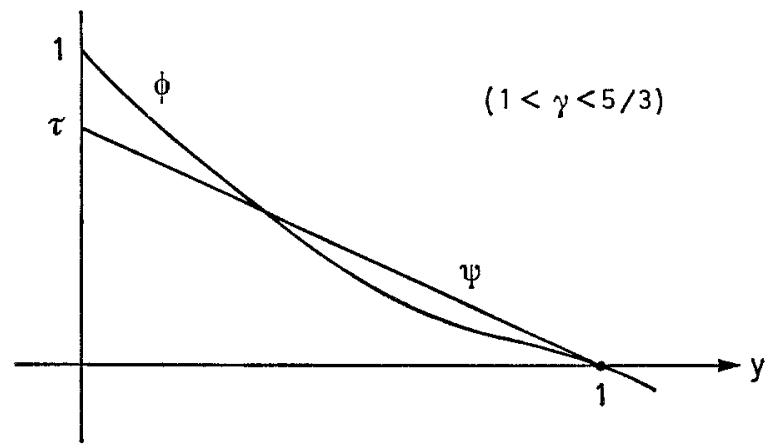

Fig. 6

Now suppose that $1<\gamma<\frac{5}{3}$; then since $Q^{\prime}(1)>0$ we see that $\phi(y)$ is concave and lies below the $\psi(y)$ on a small neighborhood, $1-\varepsilon<y<1$ (see Figure 6). It follows that $\phi(y)$ cannot cross $\psi(y)$ on the open interval $(1-\varepsilon, 1)$. On the other hand, since $Q^{\prime}(0)>0$, we see that $\phi(y)$ is convex on an interval of the form $0 \leqq y<\varepsilon$. Thus, in order to show that $\phi(y)$ crosses $\psi(y)$ exactly once in $(0,1)$, it suffices to show that $\phi$ changes from being convex to being concave only once on $0<y<1$. Showing this in turn is equivalent to showing that $Q(y)$ has only one zero on $0<y<1$. Now in view of (25), it suffices to show that $Q^{\prime}(y)$ is a convex function on $0<y<1$; cf. Figure 7. In order to do this we compute

$$
\begin{aligned}
& Q^{\prime \prime}(y)=8 \tau^{2} \beta^{2}+2 \beta^{2}-(2-\tau)(1-\tau) \beta^{2} y^{-\tau}, \\
& Q^{\prime \prime \prime}(y)=\tau(2-\tau)(1-\tau) y^{-(1+\tau)} .
\end{aligned}
$$

Thus $Q^{\prime \prime \prime}(y)>0$ on $0<y<1$ since $0<\tau \leqq \frac{1}{5}$ if $1<\gamma \leqq \frac{5}{3}$. This completes the proof in the case $1<\gamma<\frac{5}{3}$.
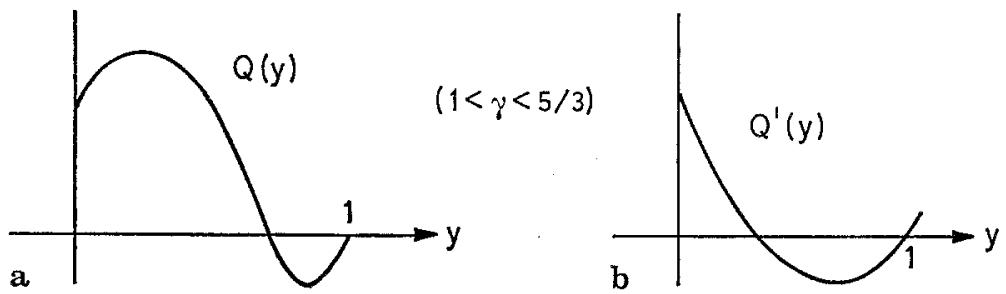

Fig. 7 

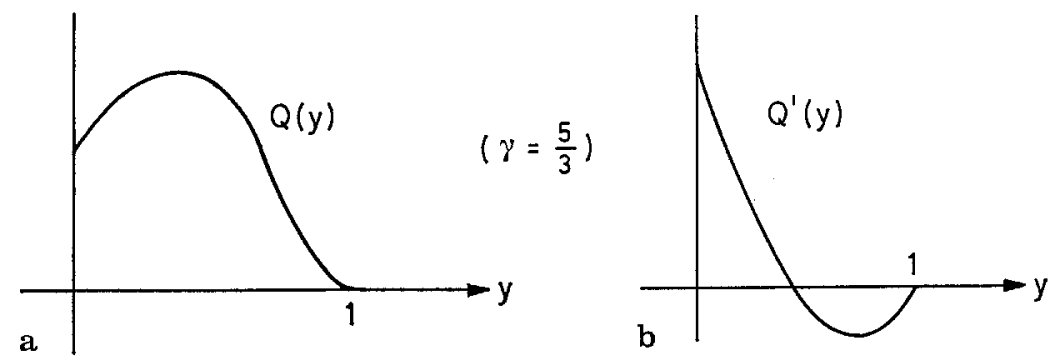

Fig. 8

Assume now that $\gamma=\frac{5}{3}$. Then as $Q^{\prime}$ is convex, $Q^{\prime}(0)>0, Q^{\prime}(1)=0$, and $Q^{\prime \prime}(1)>0$, it follows that $Q^{\prime}$ has exactly one root in $0<y<1$ (see Figure 8), so that $\phi^{\prime \prime}>0$ in $0<y<1$. But as $\phi$ is tangent to $\psi$ at $y=1, \phi$ must lie above $\psi$ on $0 \leqq y<1$. This completes the proof in this case.

This last lemma implies that the $B_{1}$ (or $\tilde{B}_{3}$ ) and $R_{1}$ (or $\tilde{R}_{\mathbf{3}}$ ) curves meet in exactly one point in the region $p<p_{L}$ (or $p<p_{r}$ ) if $1<\gamma<\frac{5}{3}$. Using this lemma, for $1<\gamma<\frac{5}{3}$, we define the number $y_{*}$ to be the unique root of (24) in $0<y<1$, i.e.,

$$
\sqrt{\bar{\tau}}\left(1-y_{*}\right)=\left(1-y_{*}^{\tau}\right)\left(1+\beta y_{*}\right)^{1 / 2}, \quad 0<y_{*}<1 ;
$$

$\beta=\frac{\gamma+1}{\gamma-1}, \tau=\frac{\gamma-1}{2 \gamma}$. Note that $y_{*}$ depends only on $\gamma, 1<\gamma<\frac{5}{3}$, and $y_{*}=\exp \left(-x_{*}\right)$ for some $x_{*}>0$. If we recall from (23) that $\exp \left(-x_{*}\right)=\bar{p} / p_{L}$, then the $p$-coordinate of the point of intersection of $B_{1}$ and $R_{1}$ satisfies

$$
\bar{p}=p_{L} y_{*} .
$$

It follows that if $(u, p) \in B_{1}\left(u_{L}, p_{L}\right)$ and $p<p_{L} y_{*}$, then the solution of the Riemann problem with data $\left(u_{L}, p_{L}, e_{L}\right)$ on the left and $(u, p, \varrho)$ on the right is solvable by a 1-rarefaction wave, a contact discontinuity and a 3-shock wave; $c f$. Figure 9. Hence for sufficiently strong rarefaction shocks, Theorem 1 does not apply.

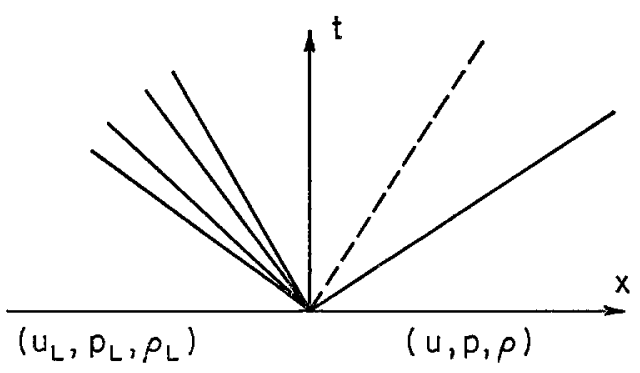

Fig. 9 


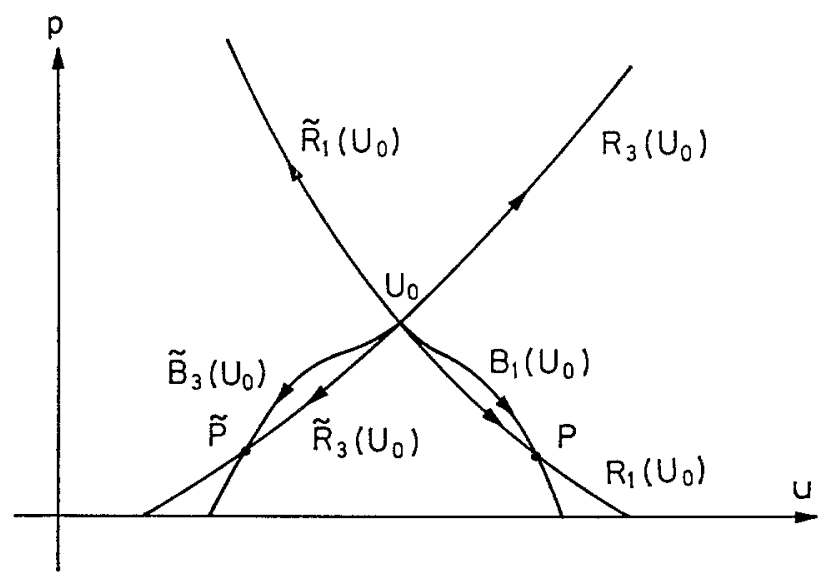

Fig. 10

We shall now clarify precisely those rarefaction shocks for which Theorem 1 is valid. To this end let $U_{0}=\left(u_{0}, p_{0}, \varrho_{0}\right)$, and denote by $R_{1}\left(U_{0}\right)$ and $B_{1}\left(U_{0}\right)$ those states which can be connected to $U_{0}$ on the right by a 1-rarefaction wave and a 1-rarefaction shock respectively. Similarly, let $\tilde{R}_{3}\left(U_{0}\right)$, and $\tilde{B}_{3}\left(U_{0}\right)$ denote the states which can be connected to $U_{0}$ on the left by a 3-rarefaction wave and a 3-rarefaction shock, respectively; $c f$. Figure 10. The (unique) crossing points of rarefaction shock curves are denoted by (cf. Figure 10) $P\left(U_{0}\right)=\left(u_{1}, p_{1}\right), p_{1}=y_{*} p_{0}$, and $\tilde{P}\left(U_{0}\right)=\left(u_{2}, p_{2}\right), p_{2}=y_{*} p_{0}$. Observe that

$$
\left.\begin{array}{l}
B_{1}\left(U_{0}\right)(p) \geqq R_{1}\left(U_{0}\right)(p) \\
\tilde{B}_{3}\left(U_{0}\right)(p) \leqq \tilde{R}_{3}\left(U_{0}\right)(p)
\end{array}\right\} \quad \text { if } y_{*} p_{0} \leqq p \leqq p_{0} .
$$

We can now state the main lemma.

Lemma 7. Let $1<\gamma<0$ and let $y_{*}$ be the unique solution of (26). Suppose that $U_{r}=\left(u_{r}, p_{r}, \varrho_{r}\right)$ can be connected to $U_{l}=\left(u_{l}, p_{l}, \varrho_{l}\right)$ on the right by a rarefaction shock. If

$$
\left|p_{r}-p_{l}\right| \leqq \begin{cases}\left(1-y_{*}\right) p_{l}, & \text { for } p_{r}<p_{l}, \\ \frac{1}{y_{*}}\left(1-y^{*}\right) p_{l}, & \text { for } p_{r}<p_{l},\end{cases}
$$

it follows that $U_{r}$ can also be connected to $U_{l}$ on the right by two rarefaction waves and (possibly) a contact discontinuity.

Proof. There are two possibilities, depending on whether $p_{r}<p_{l}$ or $p_{r}>p_{l}$ (cf. Figure 7). Suppose first that $p_{r}<p_{l}$. Then $U_{r}$ is connected to $U$ by a 1-rarefaction shock, and (29) gives $y_{*} p_{l} \leqq p_{r}<p_{l}$. It follows from (28) that the Riemann problem $\left(U_{l}, U_{r}\right)$ is resolved by connecting $U_{l}$ to $U_{r}$ by a unique 1-rarefaction wave, a contact discontinuity, and a 3-rarefaction wave.

Now suppose that $p_{r}>p_{l}$; then $U_{r}$ is connected to $U_{l}$ by a 3-rarefaction shock. Now through $U_{r}$ we draw the curves $\tilde{B}_{3}\left(U_{r}\right)$ and $\tilde{R}_{3}\left(U_{r}\right)$; then $\tilde{B_{3}}\left(U_{r}\right)$ must 


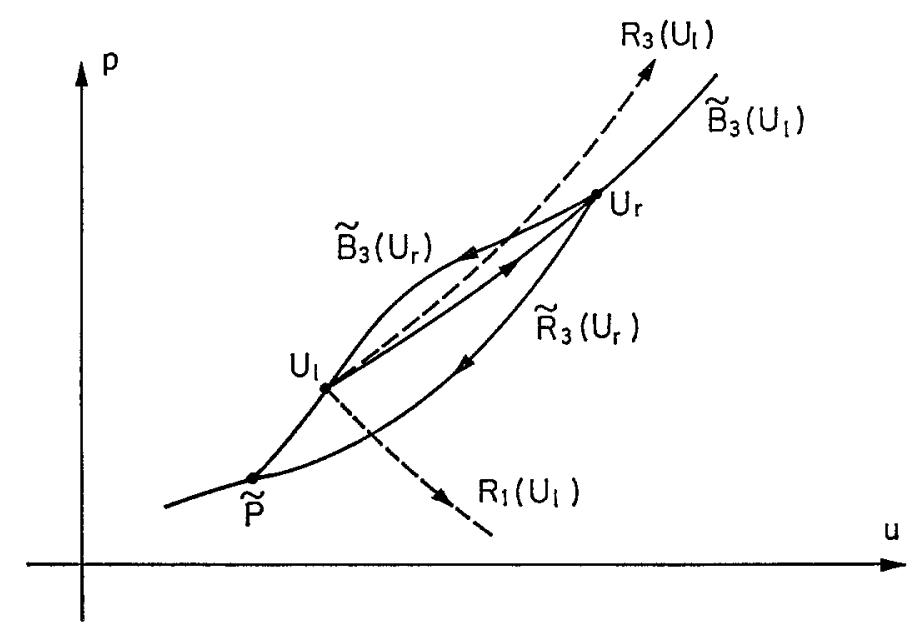

Fig. 11

go through $U_{l}$, since $U_{r} \in B_{3}\left(U_{l}\right) ; c f$. Figure 11. Thus in this case, (29) implies that $y_{*} p_{r} \leqq p_{l}<p_{r}$, so that from (28), $U_{r}$ can be connected to $U_{l}$ on the right by a 1-rarefaction wave, a contact discontinuity, and a 3-rarefaction wave. This completes the proof of the lemma.

As an immediate corollary of Theorem 1 and Lemmas 5 and 7 we have the following theorem.

Theorem 8. Let $1<\gamma<\frac{5}{3}$ and let $y_{*}$ be the unique solution of (26). Suppose that $U_{r}=\left(u_{r}, p_{r}, \varrho_{r}\right)$ can be connected to $U_{l}=\left(u_{l}, p_{l}, \varrho_{l}\right)$ on the right by a rarefaction shock. Then if (29) holds, this refraction shock is unstable in the class of smooth solutions.

We shall discuss in $\S 6$ some further implications of the above results, but first we consider a converse to Theorem 1 , in the case of two equations.

\section{§ 4. A Stability Theorem for Pairs of Conservation Laws}

In this section we consider systems of two strictly hyperbolic genuinely nonlinear conservation laws. We shall show that if the admissible solution of the Riemann problem for a given rarefaction shock contains a compressible shock wave, then the rarefaction shock (having the same data) is not unstable in the class of smooth solutions. More precisely, we have the following converse of Theorem 1 for pairs of conservation laws.

Theorem 9. Consider a strictly hyperbolic, genuinely nonlinear system of two conservation laws. Assume that $\left[u_{L}, u_{R}\right]$, the admissible solution of the Riemann 
problem (2) contains at least one shock wave. Then there does not exist a $t>0$ and a family of smooth solutions in $[0, t]$ whose data tends to the initial data (2) in some $L^{p}(\mathbb{R}), p>0$.

Proof. We refer to the well-known paper of LAx [L]. Since we are considering pairs of conservation laws, we know that there exists a coordinate system $z, w$, consisting of Riemann invariants (see [S]). We may suppose that $z$ is constant on integral curves of $\mathscr{R}_{1}$ and that $w$ is constant along the $\mathscr{R}_{2}$ integral curves. We may also assume that $z$ increases with $\lambda_{2}$ on $\mathscr{R}_{2}$, and that $w$ increases with $\lambda_{1}$ along $\mathscr{R}_{1}$. Lax's theorem states that a shock will form in a solution having initial data $\left(z_{0}(w), w_{0}(x)\right)$ if

$$
\frac{d z_{0}(\bar{x})}{d x}<0, \quad \text { or } \quad \frac{d w_{0}(\bar{x})}{d x}<0,
$$

for some $\bar{x} \in \mathbb{R}$, and that the time $\bar{t}$ that the shock forms is of the order

$$
\bar{t}=\left|\inf \frac{d w_{0}}{d x}\right|^{-1} \quad \text { or } \quad \bar{t}=\left|\inf \frac{d z_{0}}{d x}\right|^{-1} \text {. }
$$

Since $\left[u_{L}, u_{R}\right]$ contains an (admissible) shock wave of one family, then by definition,

$$
\text { either } z\left(u_{R}\right)<z\left(u_{L}\right), \quad \text { or } \quad w\left(u_{R}\right)<w\left(u_{L}\right) \text {. }
$$

Thus, if $u_{\varepsilon}(x, 0)$ are smooth functions converging to $u_{0}(x)$ in $L^{p}$ for some $p>0$, then either

or

$$
\inf _{x \in \mathbb{R}}\left\{\frac{d z_{0}^{\varepsilon}}{d x}\right\} \rightarrow-\infty \quad \text { as } \varepsilon \rightarrow 0,
$$

$$
\inf _{x \in \mathbb{R}}\left\{\frac{d w_{0}^{\varepsilon}}{d x}\right\} \rightarrow-\infty \quad \text { as } \varepsilon \rightarrow 0 .
$$

Therefore the time of blow-up of $u_{\varepsilon}(x, t)$ tends pointwise to zero as $u_{\varepsilon}(x, 0) \rightarrow u_{0}(x)$. That is, no sequence of approximating smooth solutions can be defined uniformly on any $t>0$, and thus the instability of the rarefaction shock cannot be observed within the class of smooth solutions alone. This completes the proof.

\section{§5. Concluding Remarks}

Our results in $\S 4$ show that if one considers the full gas dynamics (Euler) equations with a polytropic equation of state, in the range $1<\gamma<\frac{5}{3}$, then rarefaction shocks $\left(u_{l}, p_{l}, \varrho_{l}\right),\left(u_{r}, p_{r}, \varrho_{r}\right)$ which satisfy

or

$$
y_{*} p_{l} \leqq p_{r}<p_{l} \quad \text { for } 1 \text {-shocks, }
$$

$$
y_{*} p_{r} \leqq p_{l}<p_{r} \quad \text { for } 3 \text {-shocks, }
$$

are unstable relative to smoothing; this is a consequence of Theorem 8 . We conjecture that the same result holds for rarefaction shocks of arbitrary strength. As we have seen, this statement does not follow; from Theorem 1. 
Next, we want to point out that some care must be taken in solving Riemann problems in the $(u, p)$-plane. Indeed, sometimes the solution does not even have the expected form. We shall illustrate this by an example. Thus, assume that $U_{l}=\left(u_{l}, p_{l}, \varrho_{l}\right)$ is a given state and that $U_{r}=\left(u_{r}, p_{r}, \varrho_{r}\right)$ lies on $B_{1}\left(U_{l}\right)$, the 1rarefaction wave curve through $U_{l}$. Suppose further that $p_{r}<y_{*} p_{l}$, where $y_{*}$ is defined by (26). This geometry is depicted in Figure 12a. Note that the unique admissible solution of the Riemann problem with data $\left(U_{l}, U_{r}\right)$ consists of a 1-rarefaction wave, a contact discontinuity and a 3 -shock wave; $c f$. the discussion preceding Figure 6. Now let us consider $U_{r}$ as a given state on the right, and consider the $\tilde{R}_{1}$ and $\tilde{R}_{3}$ curves, starting at $\left(u_{r}, p_{r}\right)$, in the $(u, p)$-plane; $c f$. Figure $9 \mathrm{~b}$. According to Lemma $5 \mathrm{~b}$, the point $\left(u_{l}, p_{l}\right)$ lies in the $\tilde{R_{1}}\left(u_{r}, p_{r}\right)-\tilde{R_{3}}\left(u_{r}, p_{r}\right)$ region; i.e., it lies in the region where one "expects" to solve Riemann problems, by rarefaction waves and contact discontinuities. However, this is misleading. Indeed, even though $\left(u_{l}, p_{l}\right)$ lies in the $\tilde{R}_{1}\left(u_{r}, p_{r}\right)-\tilde{R}_{3}\left(u_{r}, p_{r}\right)$ region, the Riemann problem $\left(U_{l}, U_{r}\right)$ is not solvable by rarefaction waves and contact discontinuities, because the $R_{1}\left(u_{l}, p_{l}\right)$ curve lies above $\left(u_{r}, p_{r}\right)$. Thus it is impossible to connect

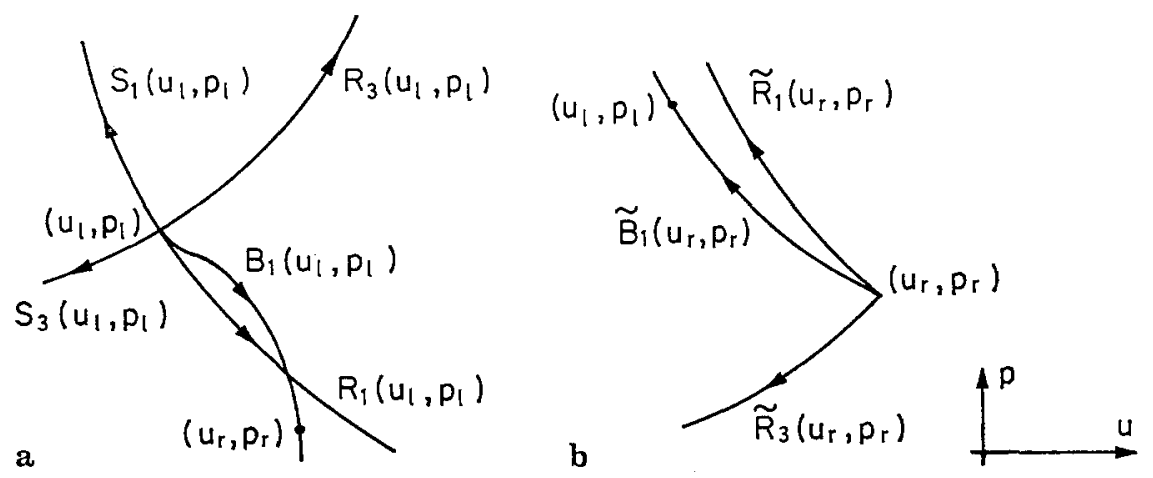

Fig. 12

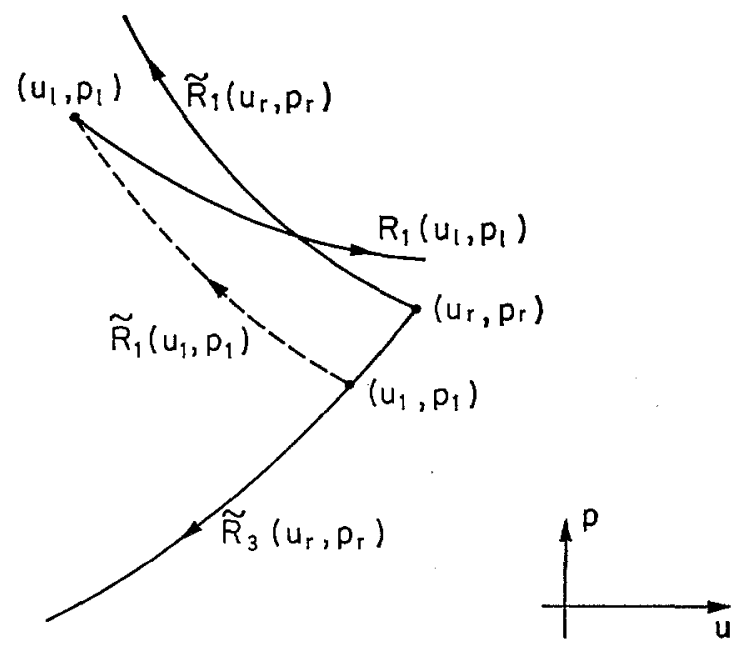

Fig. 13 
any point $P$ on $\tilde{R}_{3}\left(u_{r}, p_{r}\right)$ to $\left(u_{l}, p_{l}\right)$ by an $\tilde{R}_{1}(P)$ curve; here we are using the fact that if $\left(u_{r}, p_{r}, \varrho_{r}\right)$ lies on $R_{1}\left(u_{l}, p_{l}, \varrho_{l}\right)$, then the curves $R_{1}\left(u_{l}, p_{l}, \varrho_{l}\right)$ and $\tilde{R}_{1}\left(u_{r}, p_{r}, \varrho_{r}\right)$ coincide ( $c f$. [S, p. 363]). Thus the geometry in Figure $12 \mathrm{~b}$ is depicted with greater precision in Figure 13, and the dashed $\tilde{R}_{1}$ curve connecting $\left(u_{1}, p_{1}\right)$ is not the correct $R_{1}\left(u_{l}, p_{l}\right)$ curve; it lies on a different density level. Indeed, the apparent fallacy is traceable to the fact that one loses important information upon projecting the three-dimensional shock and rarefaction wave curves onto the $(u, p)$-plane.

Finally, we remark that in view of the recent results of Z. P. XIN [X] for two equations, any rarefaction shock is unstable in still another sense: If one considers the associated parabolic system (now modified by the addition of "viscosity" terms) and takes initial data close to the data for the Riemann problem for the rarefaction shock, then as $t \rightarrow \infty$, the corresponding solution tends not to the rarefaction shock, but rather to the solution of the Riemann problem for the hyperbolic system consisting of two rarefaction waves.

Acknowledgment. SMOLLER's work was supported in part by the NSF under grant No. MCS-830123, and in part by the ONR under grant No. N0014-88-K-0082. TEMPLE's work was supported in part by the NSF under grant No. DMS-8613450 and XIN's work was supported in part by the Rackham Predoctoral Fellowships.

\section{References}

[CS] CONWAy, E. \& J. SMOLlER, Shocks violating Lax's conditions are unstable, Proc. Amer. Math. Soc. 39 (1973), 253-256.

[CF] Courant, R. \& K. O. Friedrichs, Supersonic Flow and Shock Waves, WileyInterscience: New York, 1948.

[IMPT] Isaacson, E., D. Marchesin, B. Plohr \& B. Temple, The Riemann problem near a hyperbolic singularity: the classification of quadratic Riemann problems, I, SIAM J. Appl. Math. (to appear).

[SJ] SMoller, J. \& J. Johnson, Global solutions for an extended class of hyperbolic systems, Arch. Rational Mech. Anal. 32 (1969), 169-189.

[L] LAX, P., Development of singularities of nonlinear hyperbolic partial differential equations, J. Math. Phys. 5 (1964), 611-613.

[S] SMOLLER, J., Shock Waves and Reaction-Diffusion Equations, Springer-Verlag: New York (1983).

[T] Temple, B., Decay with a rate for noncompactly supported solutions of conservation laws, Trans. Amer. Math. Soc. 298 (1986), 43-82.

[TE] Temple, B., Stability and decay in systems of conservation laws, Proc. of First International Conference on Hyperbolic Conservation Laws, Saint Etienne, Jan. 1986.

[X] XIN, Z. P. Asymptotic stability of rarefaction waves for $2 \times 2$ viscous hyperbolic conservation laws, J. Diff. Eqs. 73 (1988), 45-77.

Department of Mathematics University of Michigan

Ann Arbor, Michigan and

Department of Mathematics University of California Davis, California 